\title{
Stereotypy of spatial movements during noncontingent and contingent reinforcement
}

\author{
FRANCISCO J. SILVA \\ Indiana University, Bloomington, Indiana \\ and \\ JOSEPH J. PEAR \\ University of Manitoba, Winnipeg, Manitoba, Canada
}

\begin{abstract}
The degree of stereotypy in the movement patterns of 3 pigeons during noncontingent and contingent periodic food reinforcement was quantified by analyzing the distribution of turning angles, and by using information and Fourier analyses. The results indicated that (1) movement patterns were less stereotyped during noncontingent than during contingent reinforcement, (2) a reversal to noncontingent reinforcement resulted in a degree of stereotypy comparable to that during the first phase of noncontingent reinforcement, (3) movement patterns were maximally stereotyped immediately after food withdrawal and generally became less stereotyped as reinforcement approached, regardless of whether reinforcement was noncontingent or contingent, and (4) higher frequency movements generally accounted for more variance in the movements during contingent than during noncontingent reinforcement. Greater stereotypy in the movements during contingent reinforcement was likely due to a greater probability that similar movements were reinforced during contingent reinforcement. Momentary changes in the stereotypy of the movements within the interfood interval might reflect changes in the level of arousal.
\end{abstract}

Repeated reinforcement, provided that it is not explicitly made contingent on behavioral variability (see, e.g., Machado, 1989, 1992, 1994; Page \& Neuringer, 1985), typically results in some degree of response stereotypy. For example, reinforcing a particular response over time reduces the variability in its force, duration, and topography, even when no explicit contingency is programmed to restrict variability (see, e.g., Antonitis, 1951; Boulanger, Ingebos, Lahak, Machado, Richelle, 1987; Eckerman \& Lanson, 1969; Guthrie \& Horton, 1946; Notterman \& Mintz, 1965; Skinner, 1938). Moreover, reinforcing a large class of behavioral sequences leads to the predominance of a few members of that class (see, e.g., Morris, 1987; Pisacreta, 1982; Schwartz, 1980, 1982; Vogel \& Annau, 1973). Finally, both contingent and noncontingent reinforcement engender repetitive sequences of behavior (see, e.g., Eldridge, Pear, Torgrud, \& Evers, 1988; Pear, 1985; Silva,

We thank Eliot Hearst, Steve Holborn, Witold Kinsner, Armando Machado, Allen Neuringer, Bob Tait, Bill Timberlake, and David Widman for their helpful suggestions and discussions, Simon Benhamou for providing the significant portions of the program that analyzed the turning angles, and William Vetterling for theoretical and technical assistance regarding information and Fourier analyses. Part of this study was used in a dissertation submitted by F.J.S. to the Faculty of Graduate Studies at the University of Manitoba. Please address correspondence to F.J. Silva, Department of Psychology, Indiana University, Bloomington, IN 47405 (e-mail: fsilva@ucs.indiana.edu) or to J.J. Pear, Department of Psychology, University of Manitoba, Winnipeg, MB R3T 2N2, Canada (e-mail: pear@ccu.umanitoba.ca).

-Accepted by previous editor, Vincent M. LoLordo
Silva, \& Pear, 1992; Skinner, 1948; Staddon \& Simmelhag, 1971; Timberlake \& Lucas, 1985).

Considerations of operant and adaptive-evolutionary accounts of behavior during noncontingent and contingent reinforcement can lead to different predictions regarding behavioral stereotypy. For instance, Skinner (1948) observed that pigeons engaged in idiosyncratic but stereotyped responses between presentations of unsignaled, noncontingent food. He termed these responses "superstitious" because the animal behaved as if there was a programmed contingency between its behavior and the occurrence of food, when no such relationship existed. However, several subsequent studies have suggested that superstitious behavior is related more to species-typical foraging responses elicited by food than to arbitrary operant behavior reinforced by the food (e.g., Allan \& Matthews, 1989, 1992; Innis, Simmelhag-Grant, \& Staddon, 1983; Staddon, 1992; Staddon \& Simmelhag, 1971; Timberlake, 1990; Timberlake \& Lucas, 1985; but see Justice \& Looney, 1990). Given that presentation of noncontingent food is procedurally more similar to Pavlovian than to operant conditioning (Staddon, 1992), and that noncontingent food likely elicits species-typical foraging responses, movements during noncontingent reinforcement might be more stereotyped than those during contingent reinforcement. As Timberlake (1983) points out, imposing a contingency may interfere with an animal's evolved feeding system, resulting in more variable behavior. In contrast, an operant account predicts that behavior will be more stereotyped during contingent than during noncontingent reinforcement because contingent reinforcement ensures that 
similar movements will be repeatedly reinforced, at least at the time reinforcement is about to occur, whereas noncontingent reinforcement (by definition) does not.

Regardless of whether reinforcement occurs contingently or noncontingently, one might also expect systematic changes in the stereotypy of the movements within an interfood interval (IFI). Craig (1918) classified the behavior of hungry pigeons into two categories: appetitive and consummatory. Although both activities are controlled by hunger, appetitive behavior is characterized by restless activity and variable movements related to searching, and it occurs when the probability of food is low. In contrast, consummatory behavior is characterized by stereotyped movements and occurs when the probability of food is high (see also Timberlake \& Silva, 1995; Wasserman, 1981). Staddon and Simmelhag (1971) made a similar distinction in what they called interim and terminal responses, where the former occur just after and the latter just before periodic noncontingent food presentations (see also Hanson, 1991). In relation to the appetitiveconsummatory distinction, interim responses are roughly synonymous with appetitive activities, and terminal responses are roughly synonymous with consummatory activities (Staddon \& Simmelhag, 1971, p. 14). Similarly, Matthews, Bordi, and Depollo (1990) examined the functional characteristics of interim and terminal responses and classified behavior during periodic noncontingent reinforcement into kinesic and taxic stereotypies. Kinesic responses result simply from periodic reinforcement, but taxic responses result from a change in the momentary probability of reinforcement. Thus, Craig's and Staddon and Simmelhag's classifications suggest that movements just before and just after reinforcement should be the most and least stereotyped, respectively, because these are the responses most and least contiguous with the back action of the appetitive stimulus. Matthews et al.'s classification suggests that movements immediately before and immediately after reinforcement should at least differ stereotypically from movements between these two extremes.

The purpose of the present study was to provide information relevant to the views mentioned above on behavioral stereotypy during noncontingent and contingent reinforcement. The degree of stereotypy in the movement patterns of 3 pigeons during noncontingent and contingent reinforcement was quantified by analyzing the distribution of turning angles and by using information and Fourier analyses. The data were collected by Eldridge et al. (1988). In that study, 3 pigeons were first exposed to a fixed-time (FT) 15-sec schedule in which food was presented every $15 \mathrm{sec}$ independently of the bird's behavior. After several sessions of FT 15-sec, the birds were exposed to a fixed-interval (FI) 15 -sec schedule in which food was presented contingent on a target response after $15 \mathrm{sec}$. The target response required the pigeon to contact a virtual sphere (a computer-defined spherical region located within the experimental chamber) with its head. After several sessions of FI 15 -sec, the birds were reexposed to FT 15-sec.
Although there are several techniques for examining the stereotypy of movement patterns (Bell, 1991; Colgan, 1978), there were logical and pragmatic reasons for analyzing the turning angles, and for using the information and Fourier analyses in the present study. In terms of logical considerations, the three measures cover a molarmolecular continuum of descriptions of behavioral stereotypy, with the analysis of the distribution of turning angles at the molar end and the Fourier analysis at the molecular end. Moreover, the three indices of behavioral stereotypy capture different aspects of behavior-changes in direction between successive steps in two-dimensional movement patterns (analysis of turning angles), predictability of spatial location given knowledge of the time in the IFI (information analysis), and amount of variance accounted for by cyclic components of linear movement (Fourier analysis). The main pragmatic reasons for the three analyses are that the present data are in a spatiotemporal form similar to that previously used in the analysis of turning angles (see, e.g., Benhamou, 1990); that information analysis has been used previously as a measure of behavioral stereotypy (see, e.g., Machado, 1989; Neuringer, 1991; Page \& Neuringer, 1985); and that the underlying assumptions and constraints of Fourier analysis are well understood mathematically (Press, Flannery, Teukolsky, \& Vetterling, 1989). Finally, well-specified and documented algorithms and computer programs are available for all three analyses.

\section{Analysis of Turning Angles}

The movement patterns of an animal can be quantified by establishing the statistical distribution of changes in direction (i.e., turning angles) between successive steps (Benhamou, 1989, 1990, 1994; Benhamou \& Bovet, 1989, 1991, 1992; Benhamou, Sauve, \& Bovet, 1990; Bovet \& Benhamou, 1988, 1991; Dejean \& Benhamou, 1993). More specifically, a movement pattern can be suitably represented as a sequence of steps with a constant length, $\mathbf{R}$, and by changes in direction between successive steps, $\alpha$. This is true of patterns created by computer simulation and patterns obtained by recording the two-dimensional $(x, y)$ position of an animal in real time. In the latter case, it is important to note that the step length is typically variable as a result of the constant sampling frequency (e.g., once every $.10 \mathrm{sec}$ ) used in the data recording process. To overcome this time dependency in the data, actual patterns are "discretized" by looking for the first point lying distance $\mathbf{R}$ from the starting point in the pattern, then looking for the next point lying distance $\mathbf{R}$ from the first point, and so on. Thus, for a given step length $\mathbf{R}$, it is possible to use the standard deviation of the angular distribution, $\sigma_{R}$, as an index of the changes in direction between successive steps. More stereotyped movement patterns will yield standard deviations closer to zero than less stereotyped patterns.

Figure 1 is a schematic illustrating the basic data used in the analysis of the distribution of the turning angles. In the figure, the animal's movement is represented as a sequence of points, defined by the $x$ and $y$ coordinates of the 


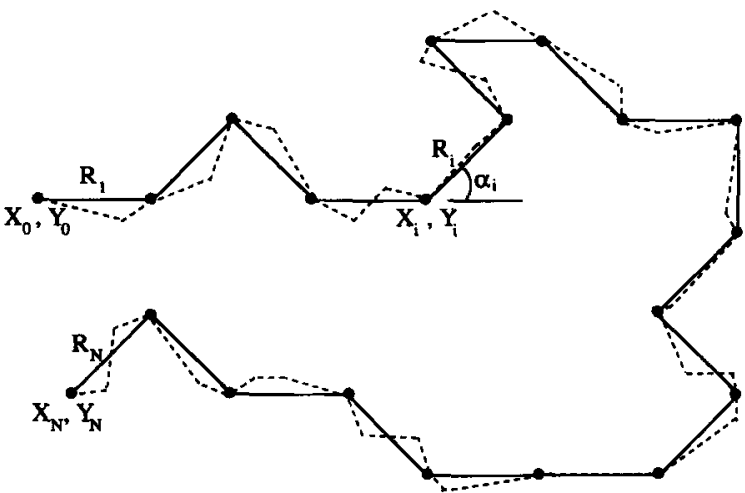

Figure 1. A schematic of a segment of a movement pattern consisting of $\boldsymbol{N}$ steps. The changes in direction (i.e., the turning angles, $\alpha$ ) are measured between adjacent steps $R_{i}$ and $R_{i+1}$. The solid and dashed lines represent the discretized and actual patterns, respectively.

animal's position relative to an origin. The distance between points is a vector with length $\mathbf{R}_{\mathbf{i}}$. The changes in direction (i.e., $\alpha$ ) are measured between adjacent vectors, $\mathbf{R}_{\mathrm{i}}$ and $\mathbf{R}_{\mathbf{i + 1}}$. The solid line shows the discretized pattern and the dashed line represents the actual pattern.

By analyzing the distribution of turning angles, one can quantify the stereotypy in any discretized movement pattern by means of a single, spatial index (Bovet \& Benhamou, 1988). With this time-independent index, it is possible to compare various patterns in terms of the standard deviation of the distribution of turning angles. It could, for example, be used to compare movement patterns produced by different species moving in the same environment or by the same species moving in different environments. For instance, an animal foraging in a patchy environment should exhibit more changes in direction (and thus the distribution of turning angles will be described by a greater standard deviation) than when it is moving between patches (Bovet \& Benhamou, 1988).

\section{Information Analysis}

Information theory has been applied to various behavioral, biological, and ecological phenomena (e.g., Altmann, 1965; Attneave, 1959; Hailman, 1982; Machado, 1989; Neuringer, 1991; Page \& Neuringer, 1985; Recuerda, Arias de Reyna, Redondo, \& Trujillo, 1987; Steinberg, 1977; Weber, Depew, \& Smith, 1988). For example, Seeley and Brozoski (1989) used an information analysis to measure the stereotypy in the movement patterns of rats injected with different doses of a stereotypy-inducing drug. In their study, stereotypy was defined as relative redundancy,

$$
R_{\text {rel }}=1-\left(H / H_{\text {max }}\right),
$$

where $H$ is the entiopy of the coordinates of an animal's position, and $H_{\max }$ is the maximum entropy if all coordinates occurred with the same frequency. Similarly, Recuerda et al. (1987) used an information analysis to quantify the degree of stereotypy in the alarm postures in red deer and concluded that an information analysis is an excellent technique for quantifying stereotypy because (1) it permits many comparisons regardless of the nature of the system, (2) continuous and discrete responses can be analyzed, and (3) a redundancy measure is intuitively simple to understand because its limits are between zero and one.

Besides being used to measure stereotypy in the movement of rats and alarm postures in deer, an information analysis can be used to answer the following question related to pigeon movements in an experimental chamber: What is the relative reduction in uncertainty about the bird's location, given knowledge of time $(t)$ in an IFI? This is a question of dependency, and it provides a measure of association between two variables (e.g., time and position). The dependency measure lies between 0 and 1 , with 0 indicating that the two variables have no association and 1 indicating that knowledge of the first variable completely predicts the second variable. Viewing $t$ as the independent variable and distance $(d)$ from some fixed object (e.g., the feeder) as the dependent variable, the dependency of $d$ on $t$ is formally defined as

$$
U(d \mid t)=[H(d)-H(d \mid t)] / H(d),
$$

where $U(d \mid t)$ is the relative reduction in uncertainty of the animal's location, given knowledge of the time in the IFI. To the extent that a movement pattern is random or variable, knowledge of the time in an IFI will provide little relative reduction in uncertainty regarding the animal's location. However, if a movement pattern is stereotyped and repeats itself across temporal intervals, then knowing the time in the IFI will provide substantial relative reduction in uncertainty about the animal's location.

\section{Fourier Analysis}

Fourier analysis is a class of computational methods used to analyze periodic processes. ${ }^{1}$ With a fast Fourier transform (FFT), it is possible to describe the amount of variation in a time series by partitioning it into sinusoids of different amplitudes and frequencies. A sinusoid is a wave whose variance can be accounted for by a single frequency (Kaplan, 1983).

An FFT fits a set of sinusoids with different amplitudes and frequencies to a time series. The degree of fit between a particular sinusoid and the time series is reflected in its contribution to the explained variance of the series, just as the importance of a linear contrast in an analysis of variance is judged by how much it accounts for the total variance. Any set of sinusoids must be orthogonal in order to carry out appropriate statistical tests of those sinusoids. For a sinusoid of a given frequency, only one other sinusoid at that same frequency is orthogonal to it-the sine perspective is orthogonal to the cosine perspective.

The variance of a time series is measured by dividing it into orthogonal sinusoids and measuring the deviations of those sinusoids from the mean of the series. The variance of the sinusoids sum to the variance of the original series, making the fraction of the time series' variance at a given frequency analogous to the proportion of variance accounted for by a pair of contrasts (except for some scale factors 
that depend on the physical system). A given frequency is analogous to a pair of contrasts because the variance at any frequency may be associated with two components in sine and cosine perspectives. A standard way of representing the amount of variance accounted for by each frequency is to construct a frequency spectrum or, if the variances are squared, a power spectrum, where the abscissa is frequency and the ordinate is "power." The time series analyzed in the present study consisted of the distance of a bird's head from the virtual target sphere sampled every $.10 \mathrm{sec}$ during several IFIs. Intuitively, it seems reasonable to assume that behavior patterns described by fewer sinusoids are more stereotyped than those that require more sinusoids in order to account for the same amount of variance.

\section{METHOD}

Because details of the subjects and experimental procedure have been given elsewhere (Eldridge et al., 1988), their redescription is brief.

\section{Subjects}

Two experimentally naive male Texas Pioneer (a White Carneau/ Silver King crossbreed) pigeons (Birds 2 and 3) were maintained at $80 \%$ of their free-feeding weights; one White King pigeon (Bird 3 ) was maintained at $90 \%$. All birds were approximately 1 year old and were housed in individual cages in a colony room regulated by a 12:12-h light:dark cycle. Water was freely available in their home cages.

\section{Apparatus}

Experimental chamber. A white metal frame supported the top and sides of the experimental chamber, the inside dimensions of which were $57 \times 57 \times 38 \mathrm{~cm}$. The front wall of the chamber was made of white opaque Plexiglas and aluminum; the left adjacent wall was made entirely of white opaque Plexiglas; the other two walls were made of clear glass; and the top cover of the chamber was made of clear Plexiglas. The floor consisted of an aluminum mesh fitted into a stainless steel drop pan. Air spaces in the top and bottom of the chamber provided ventilation to the chamber. The room lights illuminated the chamber through the top cover and the two clear glass sides, and a speaker in the room provided $82-\mathrm{dB}$ white noise as a masking stimulus.

Located on the front wall was a 6-cm-diameter feeder aperture positioned $13.5 \mathrm{~cm}$ from the floor and equidistant from the side walls. The feeder aperture was illuminated continuously; during reinforcement, however, which consisted of access to the food-filled hopper, the brightness of the feeder light was intensified.

Continuous tracking system. Tracking was done by a videoacquisition module that analyzed the signals from two black-andwhite video cameras oriented perpendicularly to each other. Discrimination between the target and the background was based on relative brightness. The cameras were electronically linked so that they scanned the observed scene synchronously at $30 \mathrm{~Hz}$. Logic circuits within the video-acquisition module determined the horizontal and vertical positions of the target in relation to each camera. A microcomputer within the video-acquisition module accepted these raw position data and used them to compute the Cartesian coordinates of the target relative to a predetermined origin. Because the videoacquisition module had limited storage capacity, a second computer (a Cromemco Z-2D) controlled the apparatus, averaged the data (yielding $10 x, y, z$ coordinates/sec), and stored the data. A block diagram of the tracking system is presented in Pear and Eldridge (1984).

\section{Procedure}

General experimental procedures. Experimental sessions were conducted at the same time daily, 5 days per week. Each session ended after 60 food presentations. After weighing, each bird had its head darkened with black shoe polish before the start of each session so that the apparatus could track the bird. Following each session, the birds were fed an amount sufficient to maintain their weights at approximately $80 \%$ (Birds 1 and 2) or $90 \%$ (Bird 3) of their free-feeding level.

Experimental design. Following feeder training, the birds were given a baseline session of $3,600 \mathrm{sec}$, during which no reinforcement was presented. They were then exposed to the following phases: (1) FT 15-sec, (2) shaping to contact the virtual target sphere, (3) gradually increasing FI values until FI 15-sec was reached, (4) food contingent upon contacting the virtual sphere with the head on FI 15sec, and (5) return to FT 15 -sec.

The computer defined a 3-cm-diameter virtual target sphere whose center was located $13.5 \mathrm{~cm}$ from the left wall, $21 \mathrm{~cm}$ from the back wall, and $32 \mathrm{~cm}$ from the aluminum mesh floor of the chamber. According to Pear and Legris's (1987) shaping method, the computer detected the position of the bird's head, and then required the bird to move at least $1 \mathrm{~cm}$ closer to the target sphere. For every 10sec period in which the bird did not move close enough to contact this new criterion, the criterion was relaxed by $0.25 \mathrm{~cm}$. This process repeated itself until the bird contacted the target sphere. Each contact of the bird's head with the target sphere resulted in the occurrence of a feedback click. Food was presented for $5 \mathrm{sec}$.

\section{Quantitative Analyses}

General procedures. Eldridge et al. (1988) simply plotted their raw data, whereas in the present study the degree of stereotypy in the movement patterns was quantified by analyzing the distribution of turning angles, and by using an information and a Fourier analysis. The last three sessions for each bird across three phases (FT 15-sec, FI 15 -sec, and reversal to FT $15-\mathrm{sec}$ ) were examined. Furthermore, the first three sessions of the reversal to FT 15 -sec were examined to identify changes in movement patterns during the transition from FI 15-sec to FT 15-sec.

Analysis of turning angles. A program based on Bovet and Benhamou's (1988) was used to analyze the distribution of turning angles. All movement patterns were discretized with a constant step length (i.e., R) of $4 \mathrm{~cm}$. Other step lengths between $1 \mathrm{~cm}$ and $8 \mathrm{~cm}$ produced comparable results. The $x, y$ coordinate values ( $10 \mathrm{val}-$ $\mathrm{ues} / \mathrm{sec}$ ) were used to calculate the standard deviation of the distribution of turning angles in the movement patterns from each IFI during the FT and FI schedules. Standard deviations of each distribution were averaged and plotted across blocks of six IFIs.

Information analysis. To describe how the birds' movement changed during the IFI, an information analysis was conducted across successive 3-sec bins. A program based on Press et al.'s (1989) algorithm was used for this analysis (see also Vetterling, Teukolsky, Press, \& Flannery, 1989). Essentially, the information analysis counted how often a bird was at a certain distance from the feeder at a certain time during 3-sec bins (i.e., the dependency of distance on time). The start of each 3-sec bin was $0,3,6,9$, or $12 \mathrm{sec}$ within an IFI. All intervals were analyzed with 0.1 -sec resolution, and the distances were analyzed to a $1-\mathrm{cm}$ resolution. Thus, we arranged a distance $\times$ time matrix in which each cell contained the sum of how often a bird occupied a particular distance at a particular time during a 3-sec bin (a $60 \times 30$ matrix, since the farthest distance the bird could occupy from the feeder was $60 \mathrm{~cm}$ and there were $3 \mathrm{sec}$ [in 0.1-sec resolution] in a time sample).

Fourier analysis. A Fourier analysis program based on Crandall and Colgrove's (1986) algorithm was used to analyze the data. In generating a spectrum from a time series, three constraints of the FFT need to be considered. First, the interpretation of the spectrum is affected by the length and starting point of the data series to be 
transformed. Second, the computational requirements of the FFT require that the number of data points used to calculate a spectrum be an integer power of 2 (i.e., sample lengths of $2,4,8,16,32$, etc., data points). Third, for a given sample length, the maximum number of cyclic components that can be determined is equal to one half the sampling rate (i.e., the Nyquist limit). For example, if the sampling rate is 600 times per minute, then, in a 1 -min period, it is possible to assess the amount of variance accounted for by frequencies from 1 through $300 \mathrm{cycles} / \mathrm{min}$. Thus, the resolution of the spectrum is determined by the number of data points in the series, to the nearest integer power of 2 , and the rate at which they were sampled. Given these considerations, $25.6 \mathrm{sec}$ of real-time data (256 data points) of the bird's distance from the target sphere were used to calculate each spectrum. Two hundred and fifty-six data points corresponded to the first $15 \mathrm{sec}$ from selected IFIs plus the first $10.6 \mathrm{sec}$ from the next IFI. The following IFIs from each session were analyzed, where a plus sign ( + ) denotes that more than one IFI was used to construct a spectrum: $1+2,7+8,13+14,19+20,25+26,31+32,37+38$, $43+44,49+50$, and $55+56$. We chose to analyze the data of the birds' distance from the target sphere rather than the feeder to provide a more complete account of the birds' behavior during the experiment without having to present data from each analysis relative to both locations.

Three mathematical transformations were performed to produce more easily interpretable spectra. First, a trigonometric transformation was performed on the original time series data to minimize the effect of fitting multiple frequencies to the same set of data points. An example will clarify the basis for this transformation. Consider a time series whose variation is mostly described by a sinusoid with a frequency of 30 cycles $/ \mathrm{min}$. The same time series would also be well described by sinusoids with frequencies of 29 and 31 cycles/ $\mathrm{min}$. Although a sinusoid with a frequency of $30 \mathrm{cycles} / \mathrm{min}$ accounts for the largest portion of the variance, the power attributed to this frequency may be underestimated because of the apparent contributions of adjacent frequencies to the power spectrum. This effect is commonly referred to as leakage (i.e., power leaks to the adjacent frequencies). To improve the estimates of dominant frequencies, trigonometric transformations are used to produce an estimate of the best-fitting sinusoid and to reduce the identification of spurious power to adjacent frequencies (see Press et al., 1989, pp. 466-472; for a discussion of the relative strengths and weaknesses of the available transformations). Second, after the Fourier transformation, the resulting amplitudes at each frequency were squared, generating a power spectrum, to accentuate the more important frequencies and minimize the less important ones. Third, the power at each frequency was scaled to a percentage of a time series' variance (or power) accounted for by that frequency. This allowed comparisons between birds and experimental phases independently of a bird's range of movement (the scale factor dependent on the physical system mentioned in the introduction).

\section{RESULTS}

\section{Topographical Analysis of Movement Patterns}

To provide a behavioral context in which to place the results of the present study, we will first briefly describe Eldridge et al.'s (1988) results. In their study, during the first exposure to FT 15-sec, all 3 birds engaged in a pattern of feeder-wall-directed behavior with occasional looping excursions from the feeder immediately after reinforcement. During FI 15 -sec, all birds performed the arbitrary operant response, which consisted of contacting the virtual target sphere near the rear of the experimental chamber, and did not engage in feeder-wall-directed behavior. During the reversal to FT $15-\mathrm{sec}$, the birds developed a be- havior sequence consisting of moving in the direction of the target sphere after reinforcement, followed by feederwall-directed behavior prior to the next reinforcement.

\section{Analysis of Turning Angles}

Figure 2 shows the standard deviation of the distribution of turning angles averaged and plotted across blocks of six successive IFIs. The birds showed (1) greater stan-
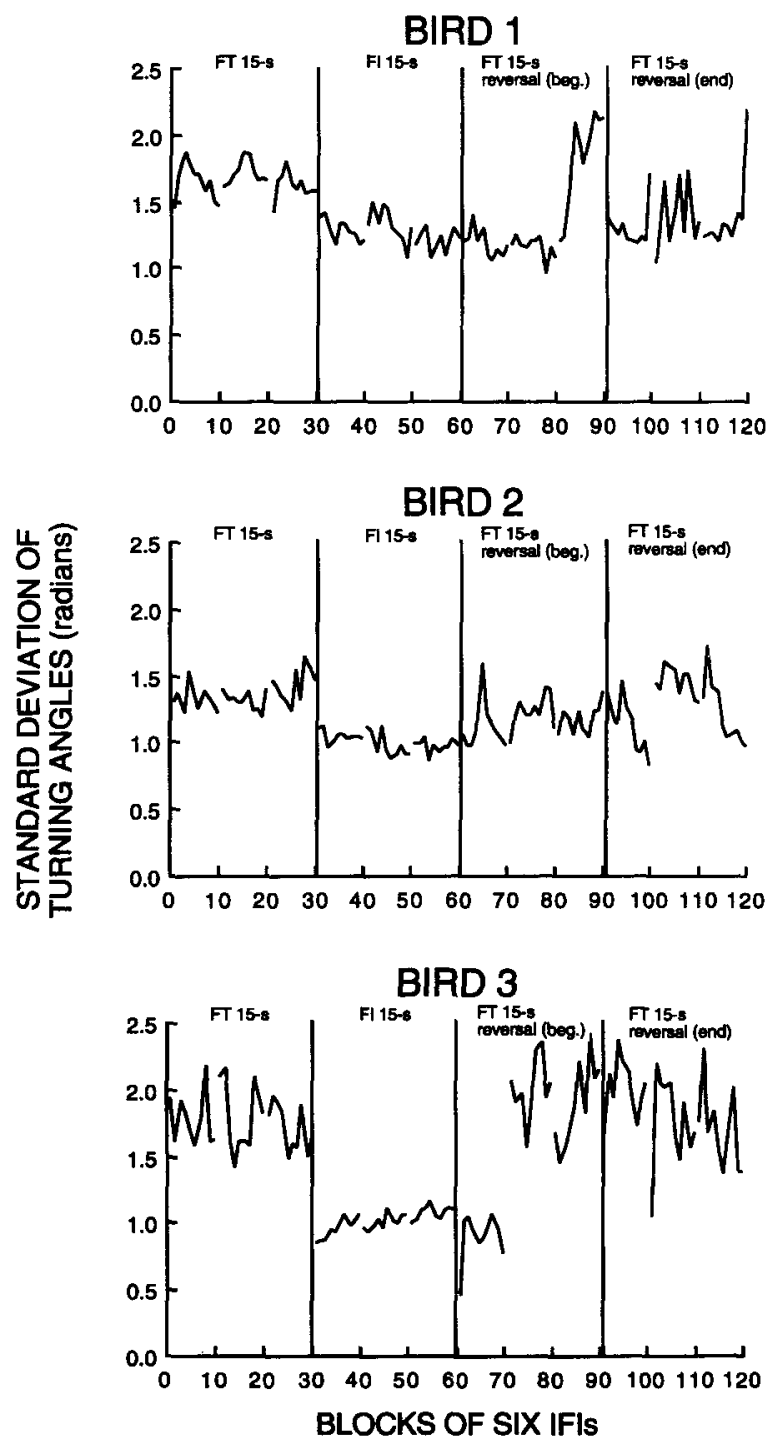

Figure 2. Standard deviations of the distributions of turning angles averaged and plotted across blocks of six interfood intervals (IFIs) for each bird. The last three sessions of fixed-time (FT) 15-sec, fixed-interval (FI) 15-sec, and the reversal to FT 15sec (FT 15-sec reversal [end]), and the first three sessions of the reversal to FT 15-sec (FT 15-sec [beginning]) are shown. There are 10 blocks per session. Different sessions are denoted by breaks in the data within a phase. The standard deviation for the movements in Block 70 of Bird 3's data could not be calculated because of insufficient movements. An increase in standard deviation indicates an increase in the changes in direction between successive steps in a movement pattern. Changes in direction are expressed in radians. 
dard deviations during FT 15-sec than during FI 15-sec, (2) more within-session consistency during FI 15-sec than during FT 15-sec, (3) a gradual (or at least delayed) increase in standard deviation when the schedule of food was changed from FI 15-sec to FT 15-sec, and (4) standard deviations in the last three sessions of the reversal to FT 15-sec comparable to those established during the first FT 15-sec phase. Furthermore, the data were more variable in the last three sessions of the reversal to FT $15-\mathrm{sec}$ than during the first FT 15-sec phase.

\section{Information Analysis}

Figure 3 indicates how stereotypy changed within the IFI by showing the dependency of distance on time or, alternatively, the relative reduction in uncertainty regarding a bird's distance from the feeder given knowledge of the time in the IFI. The data are plotted across 3-sec bins within the IFI. The figure shows the following effects: (1) dependency during the first FT 15-sec phase was less than during FI 15 -sec, (2) dependency was greatest during the first 3 -sec bin and generally decreased during the rest of the IFI, and (3) dependency during the reversal to FT 15 -sec seems to have been affected by the levels established during the previous phases.

To provide an indication of how the data in Figure 3 are related to behavior, Figure 4 shows each bird's mean distance from the feeder plotted across $3-\mathrm{sec}$ bins. The target sphere was located approximately $35 \mathrm{~cm}$ from the feeder. The following can be seen in the figure: (1) all birds were closer to the feeder during FT $15-\mathrm{sec}$ than during FI 15 -sec, (2) the birds generally were closest to the feeder during the last 3-sec bin of FT 15-sec, (3) all birds were closest to the feeder during the first 3-sec bin of FI 15-sec and farthest from the feeder during the last $3-\mathrm{sec}$ bin (when, as required by the contingency, they were near the target sphere), and (4) 2 of the 3 birds (Birds 1 and 2) spent the early part of the IFI farther from the feeder during the last three sessions of the reversal to FT 15 -sec than they did during the first FT 15-sec phase.

\section{Fourier Analysis}

Figure 5 shows power spectra for all birds with reference to the target sphere. Each graph displays the average spectrum from thirty 25.6 -sec spectra created from the samples and sessions listed in the Method section, and provides information describing which aspect of the birds' movements changed during FI 15 -sec to produce an increase in stereotypy relative to FT $15-\mathrm{sec}$. Examining the spectra from the first FT 15 -sec phase shows that all birds' movements are best characterized by a frequency of approximately 4 cycles $/ \mathrm{min}$. Reading the spectra from lower to higher frequencies, it is unclear to what extent the power at one frequency leaked from an adjacent frequency; thus, we adopted a conservative rule of thumb and assumed that any "spike" adjacent to a higher spike contained leaked power. For example, we assumed that spikes at approximately $7 \mathrm{cycles} / \mathrm{min}$ leaked from $4 \mathrm{cycles} / \mathrm{min}$ and therefore further assumed that there was no distinct movement pattern corresponding to 7 cycles $/ \mathrm{min}$. How-
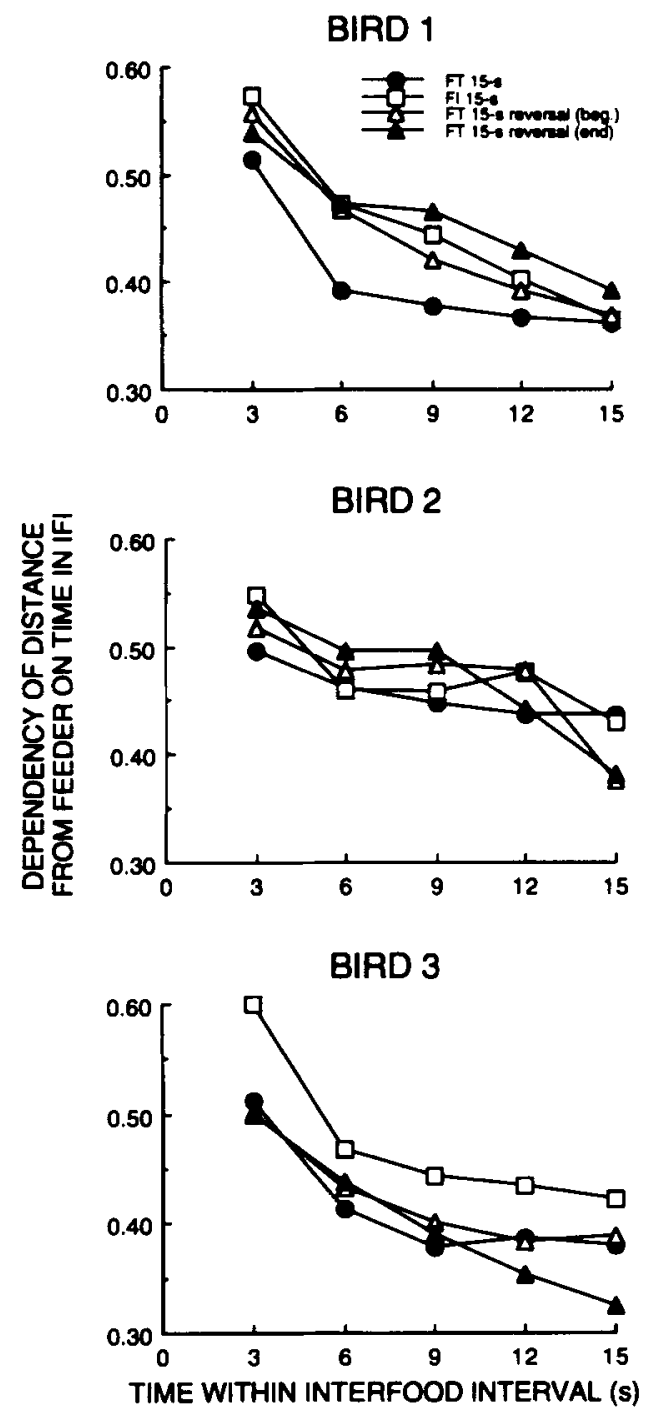

Figure 3. Reduction in relative uncertainty about a bird's distance from the feeder as a function of knowing the time in the interfood interval (IFI). Data are plotted across 3-sec bins. The last three sessions of fixed-time (FT) 15-sec, fixed-interval (FI) 15-sec, and the reversal to FT 15-sec (FT 15-sec reversal [end]), and the first three sessions of the reversal to FT 15-sec (FT 15-sec |beginning|) are shown. In the terminology of information theory, this rigure depicts the dependency of distance from the feeder on time in the IFI.

ever, the next highest spike corresponds to approximately $11 \mathrm{cycles} / \mathrm{min}$ for Birds 1 and 3 , and we assumed that these birds routinely engaged in movements that occurred approximately 11 times $/ \mathrm{min}$.

During FI 15-sec, as in the previous phase, all birds' movements were best characterized by a frequency of approximately 4 cycles $/ \mathrm{min}$. Applying the rule of thumb described above, a close examination of the data shows that, in comparison to FT $15-\mathrm{sec}$, higher frequency movements accounted for a greater percentage of the variance in all birds' movements. This is especially evident for Bird 2 at 

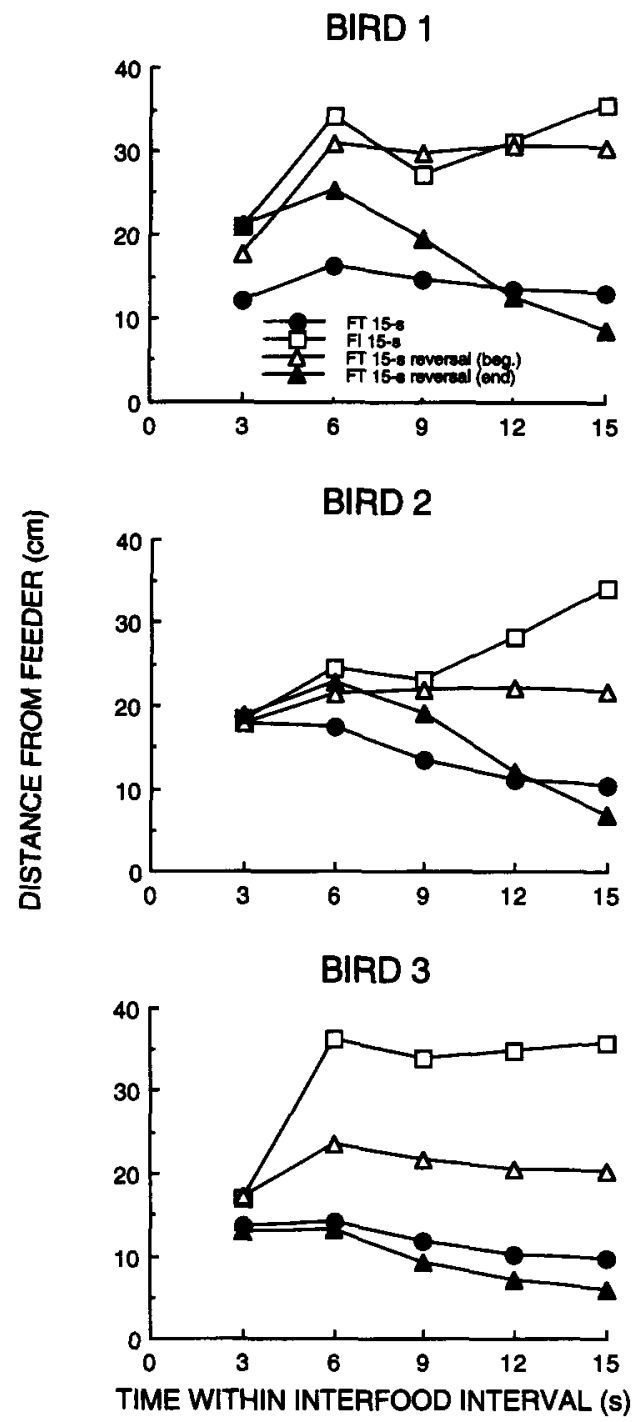

Figure 4. Mean distance of a bird from the feeder plotted across 3-sec bins. The last three sessions of fixed-time (FT) 15-sec, fixed-interval (FI) 15-sec, and the reversal to FT 15-sec (FT 15-sec reversal [end]), and the first three sessions of the reversal to FT 15-sec (FT 15-sec [beginning]) are shown.

the 11 cycles/min frequency and for Bird 3 at the 16 cycles/ min frequency, but less so for Bird 1 at the 16 cycles $/ \mathrm{min}$ frequency.

During the first three sessions of the reversal to FT 15 -sec, all birds' movements continued to be best characterized by a frequency of approximately 4 cycles $/ \mathrm{min}$, and all birds' spectra show a transition from the frequencies that characterized their movements during FI 15-sec. Bird 1's spectrum shows an enhancement of the higher 16 cycles/min frequency that began accounting for a greater percentage of the variance during FI 15-sec. Bird 2's spectrum is similar to its spectrum during the first FT 15-sec phase. Bird 3's spectrum shows a marked enhancement in the amount of variance accounted for by movements occurring at $4 \mathrm{cycles} / \mathrm{min}$, and a diminishing of the con- tribution of higher frequency movements to the explained variance.

During the last three sessions of the reversal to FT 15-sec, all birds' movements continued to be best characterized by a frequency of approximately $4 \mathrm{cycles} / \mathrm{min}$. Furthermore, the spectra generally resemble those from the first FT 15 -sec phase, except that the 4 cycles/min frequency accounts for more variance than it did during the first exposure to FT 15-sec.

In summary, movement occurring at a frequency of approximately 4 cycles/min accounted for the greatest amount of variance in the movements in all phases. Higher frequency movements accounted for more variance during FI 15-sec than during the FT 15-sec phases. Thus, it seems that the increased stereotypy during FI 15 -sec quantified by the analysis of turning angles and the information analysis was partially the result of higher frequency movements' becoming more salient (i.e., accounting for a greater percentage of the total variance) even though lower frequency movements (e.g., 4 cycles/min) continued to account for most of the variance.

\section{DISCUSSION}

The results from the three analyses conducted in this study indicate that (1) movement patterns were less stereotyped during noncontingent than during contingent reinforcement, (2) a reversal to noncontingent reinforcement following contingent reinforcement resulted in a degree of stereotypy comparable to that observed initially, (3) movement patterns were maximally stereotyped immediately after food withdrawal and generally became less stereotyped as reinforcement approached, regardless of whether reinforcement was contingent or noncontingent, and (4) higher frequency movements generally accounted for more variance in the movements during contingent than during noncontingent reinforcement.

The present data confirm past findings that reinforcement results in stereotyped behavior even when the contingency does not demand it (Machado, 1993; Notterman \& Mintz, 1965; Schwartz, 1980, 1981; Skinner, 1938; Vogel \& Annau, 1973). The finding that contingent reinforcement produced greater stereotypy than did noncontingent reinforcement is consistent with a traditional operant account, which suggests that repeated reinforcement of similar movements increases stereotypy. In contrast, an adaptive-evolutionary account that views noncontingent food as eliciting species-typical foraging responses might have led one to expect more stereotypy during noncontingent reinforcement. Imposing a contingency, especially one as seemingly unnatural as contacting a virtual sphere, might have increased variability by interfering with an animal's feeding system. In the present study, this interference did not occur.

In addition to an adaptive-evolutionary account, two other arguments might have led to a prediction of decreased stereotypy during contingent reinforcement. One argument is that the IFI is more variable during the FI schedule than during the FT schedule, owing to variation 

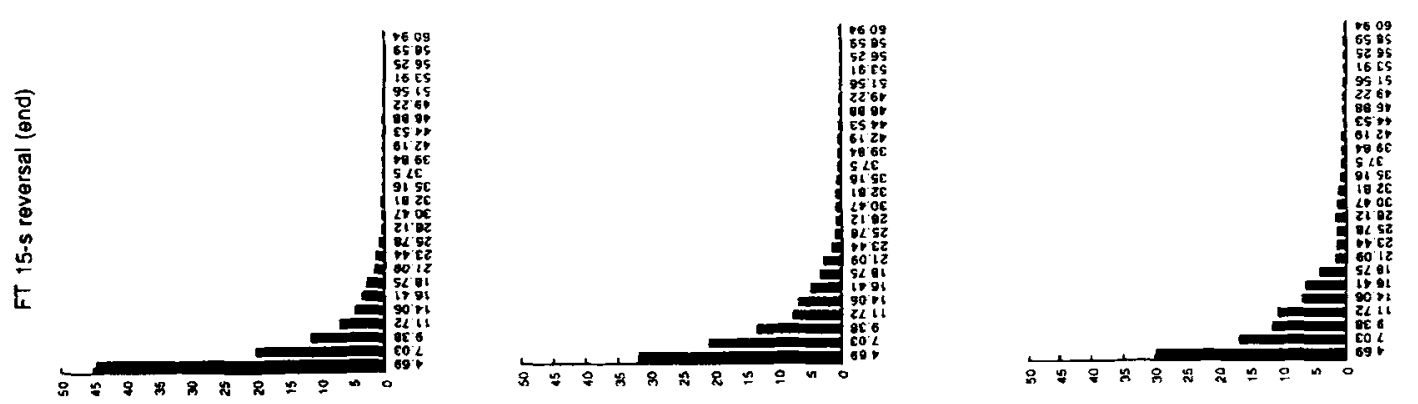

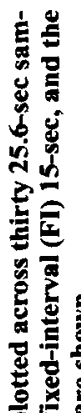

列
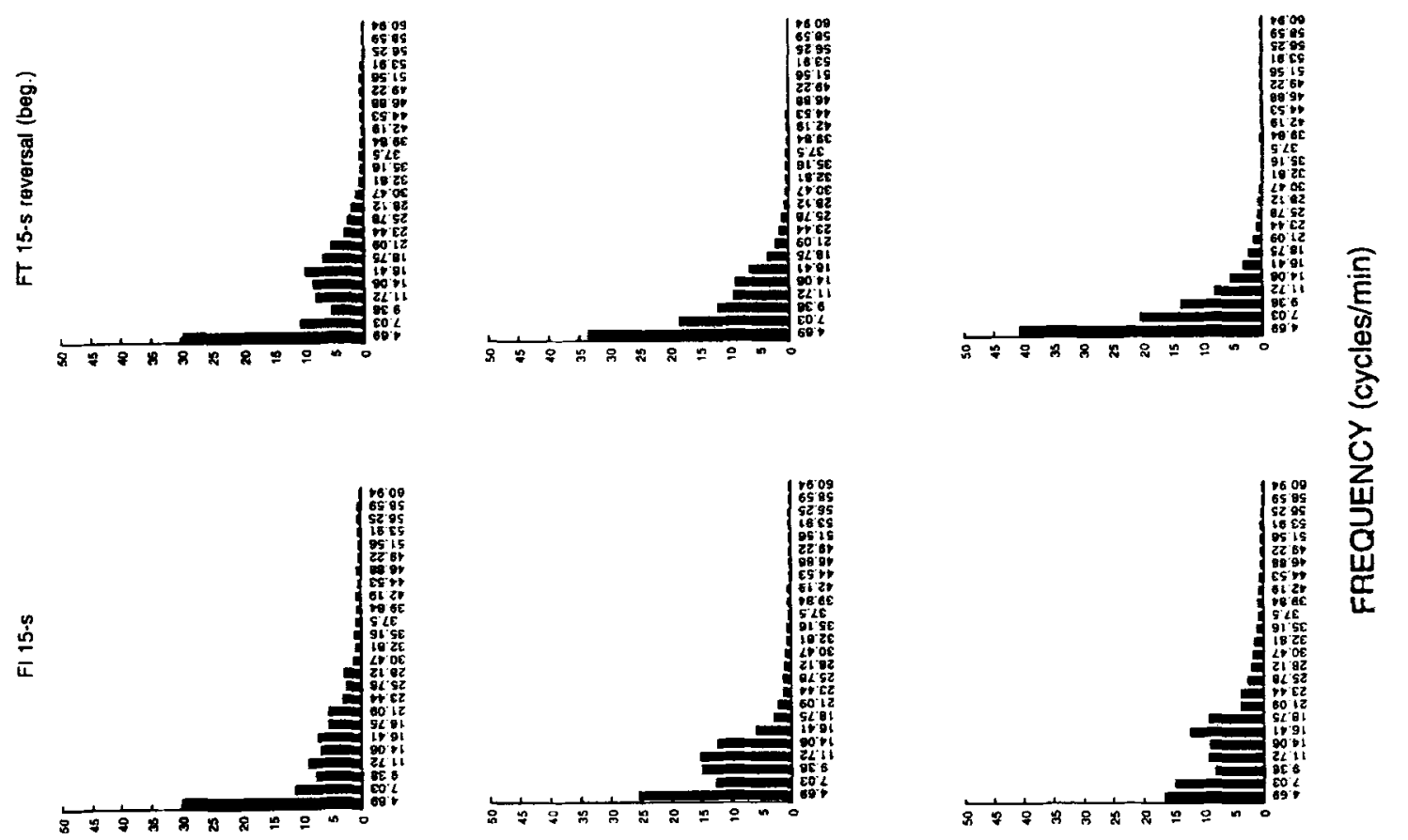

.
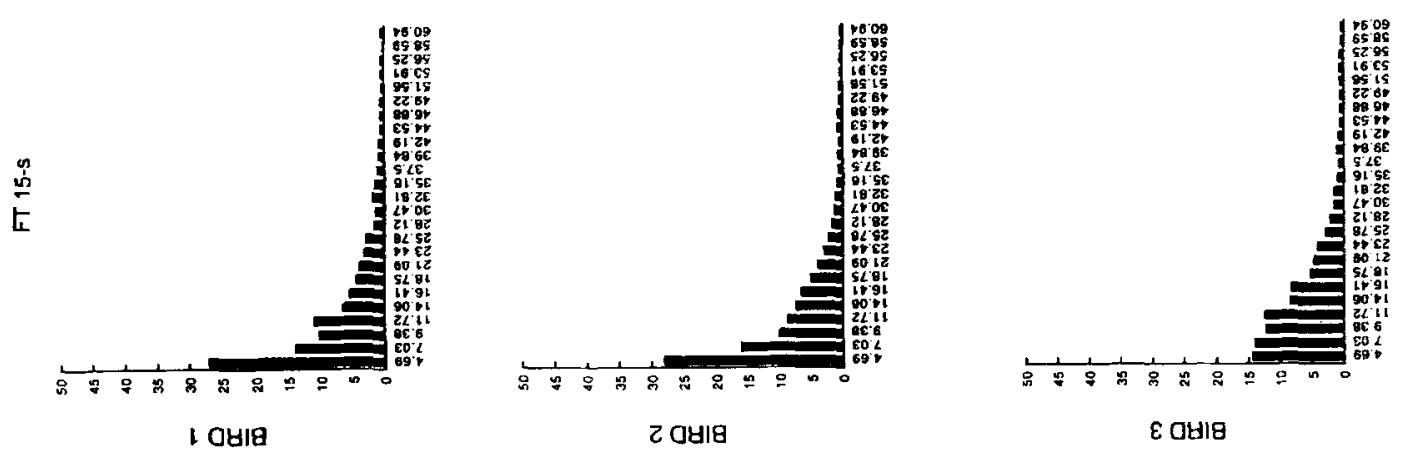

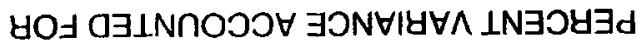


in the time at which responses occur after the time that reinforcement becomes available in FI schedules. A second argument is that, during FT, subjects might tend to move quickly to the feeder near the end of the interval (see, e.g., Timberlake \& Lucas, 1985), which might engender a high degree of stereotypy. Nevertheless, stereotypy was higher during contingent reinforcement, again supporting an operant account.

An explanation for the finding that movement patterns were most stereotyped immediately after food withdrawal and generally became less stereotyped as the time for reinforcement approached during both contingent and noncontingent food is less straightforward. Craig's (1918) appetitive-consummatory distinction and (to some extent) Staddon and Simmelhag's (1971) interim-terminal distinction suggest that movements immediately before and after reinforcement should have been the most and least stereotyped, respectively, because these are the movements most and least contiguous with the back action of reinforcement. Matthews et al.'s (1990) kinesic-taxic distinction suggests that movement patterns just before and just after reinforcement should have been stereotypically different from movements between these two extremes. However, our results did not fit with any of these views.

It may be important to note that behavioral stereotypy is often defined by observing the frequency of different categories of responses (e.g., pecking, pacing, and preening in pigeons; pressing different levers in rats) across bins within an IFI. A reduction in the diversity of a set of responses reflects increased stereotypy. For example, Hanson (1991) observed a decrease in the diversity of responses performed as the next reinforcement approached, such that a single response predominated before reinforcement (see also Timberlake \& Lucas, 1985). Similarly, Neuringer (1991) noted that a sequence of left-right leverpresses in rats became more stereotyped as the time for the next reinforcement approached. The reason for this difference between Hanson's and Neuringer's outcomes and those of our study may have to do with the different ways in which stereotypy was measured. Hanson's and Neuringer's measures were based on a reduction in the diversity of discrete response categories, whereas we used a continuous behavioral category. Changes in responses across discrete categories such as pecking or pacing may not correlate with changes along a measure of continuous movement, especially given the necessary arbitrariness of the various categories and measures used (Pear, 1994). The relationship between continuous movement and discrete behavioral categories is further complicated by the fact that, at least in rats, different behavioral categories (e.g., leverpresses, key pushes, wire pulls) generate different levels of response sequence stereotypy (Morgan \& Neuringer, 1990).

Assuming the validity of the present measure of stereotypy across IFIs, we need to address why stereotypy was greatest immediately following food withdrawal and generally decreased as the next reinforcer approached. An ex- planation based on arousal may provide an answer. Low levels of arousal are correlated with increased stereotypy (Mason, 1991a, 1991b); therefore, the general decrease in stereotypy throughout the IFI might be the result of a general increase in arousal (but see Killeen, 1975, 1979). An increase in arousal, however, is no guarantee of decreased behavioral stereotypy. Additional physiological factors, such as frustration or the presence of drugs such as alcohol, likely interact with arousal to determine stereotypy (McElroy \& Neuringer, 1990; Terlouw et al., 1991).

The Fourier analysis showed that the predominant movement patterns during contingent and noncontingent reinforcement were best characterized by a frequency of approximately 4 cycles $/ \mathrm{min}$. Given that food was presented every $15 \mathrm{sec}$ during FT and approximately every $15 \mathrm{sec}$ during $\mathrm{FI}$, it is likely that this frequency corresponded to the birds' moving toward the feeder when food was presented and moving away from the feeder soon afterward (see Figure 4, and summary of Eldridge et al.'s, 1988 , results). The higher frequencies that occurred likely corresponded to back-and-forth pacing along the feeder wall during noncontingent reinforcement (see the summary of Eldridge et al.'s, 1988, results; see also Allan \& Matthews, 1989; Matthews et al., 1990; Timberlake \& Lucas, 1985) and to pacing near the target sphere during contingent reinforcement (see Figure 4). The increased prominence of higher frequency movements during contingent reinforcement may be the birds' way of maximizing the immediacy of reinforcement by contacting the target sphere as soon as the reinforcer becomes available (Silva, Pear, Tait, $\&$ Forest, in press).

Overall, the outcome of the present study suggests that an analysis of the distribution of turning angles, an information analysis, and a Fourier analysis are suitable methods for quantifying the stereotypy of movement patterns. However, the three analyses are not necessarily interchangeable; they provide different information, ranging from a molar (analysis of turning angles) to a molecular (Fourier analysis) description. The analysis of the distribution of turning angles requires two spatial dimensions ( $x, y$ coordinates) and measures the time-independent changes in direction between successive steps in a movement pattern; the information analysis measures the dependency between two variables (distance and time); and the Fourier analysis provides information about a time series by fitting sinusoids of different frequencies to the original time series. In addition, the Fourier analysis provides some information about which aspects of the behavior have changed to permit changes in stereotypy.

Finally, the results suggest that an analysis of the distribution of turning angles can profitably be used to quantify movement patterns that occur in a relatively small enclosure such as an experimental chamber. Given that the analysis of turning angles has been applied to various facets of animal movements in natural environments, its use in the laboratory might provide another link between naturalistic and laboratory research. 


\section{REFERENCES}

Allan, R. W., \& Matthews, T. J. (1989). Comparative effects of food and water deprivation on movement patterns in the pigeon (Columba livia). Behavioural Processes, 20, $41-48$.

Allan, R. W., \& Matthews, T. J. (1992). Selective sensitivity of schedule-induced activity to an operant suppression contingency. Journal of the Experimental Analysis of Behavior, 58, 471-483.

Altmann, S. A. (1965). Sociobiology of rhesus monkeys: II. Stochastics of social communication. Journal of Theoretical Biology, 8, 490552 .

ANTONITIS, J. J. (1951). Response variability in the white rat during conditioning, extinction, and reconditioning. Journal of Experimental Psychology, 42, 273-281.

ATTNEAVE, F. (1959). Applications of information theon to psychology: A summary of basic concepts, methods, and results. New York: Holt.

BELL, W. J. (1991). Searching behaviour: The behavioral ecology of finding resources. London: Chapman \& Hall.

BENhAMOU, S. (1989). An olfactory orientation model for mammals' movements in their home ranges. Journal of Theoretical Biologv, 139 , 379-388.

Benhamou, S. (1990). An analysis of movements of the wood mouse Apodemus sylvaticus in its home range. Behavioural Processes, 22. 235-250.

Benhamou, S. (1994). Spatial memory and searching efficiency. Animal Behaviour, 47, 1423-1433.

Benhamou, S., \& Bovet, P. (1989). How animals use their environment: A new look at kinesis. Animal Behaviour, 38, 375-383.

Benhamou, S., \& Bovet, P. (1991). Modeling and simulation of animal movements. In J. Meyer \& S. W. Wilson (Eds.), From animals to animats: Proceedings of the first international conference on simulation of adaptive behavior (pp. 135-139). Cambridge, MA: MIT Press.

Benhamou, S., \& Bovet, P. (1992). Distinguishing between elementary orientation mechanisms by means of path analysis. Animal Behaviour, 43, 371-377.

Benhamou, S., Sauve, J.-P., \& Bovet, P. (1990). Spatial memory in large scale movements: Efficiency and limitation of the egocentric coding process. Journal of Theoretical Biology, 145, 1-12.

BLOOMFIELD, P. (1976). Fourier analysis of time series: An introduction. New York: Wiley.

Boulanger, B., Ingebos, A., Lahak, M., Machado, A., \& RiCHELLE, M. (1987). Variabilité comportementale et condionnement operant chez l'animal [Behavioral variability and operant conditioning in animals]. L'Année Psychologique, 87, 417-434.

Bovet, P., \& Benhamou, S. (1988). Spatial analysis of animal's movements using a correlated random walk model. Journal of Theoretical Biology, 131, 419-433.

Bovet, P., \& Benhamou, S. (1991). Optimal sinuosity in central place foraging movements. Animal Behaviour, 42, 57-62.

BrockWelL, P. J., \& Davis, R. A. (1987). Time series: Theory and methods. New York: Springer-Verlag.

Colgan, P. W. (1978). Quantitative ethology. New York: Wiley.

CraIG, W. (1918). Appetites and aversions as constituents of instincts. Biological Bulletin, 34, 91-107.

Crandall, R. E., \& Colgrove, M. M. (1986). Scientific programming with Macintosh Pascal. New York: Wiley.

DejEan, A., \& Benhamou, S. (1993). Orientation and foraging movements in a patchy environment by the ant (Serrastruma lujae) (formicidae-myrmicinae). Behavioural Processes, 30, 233-244.

ECKERMAN, D. A., \& LANSON, R. N. (1969). Variability of response location for pigeons responding under continuous reinforcement, intermittent reinforcement, and extinction. Journal of the Experimental Analysis of Behavior, 12, 73-80.

Eldridge, G. D., Pear, J. J., Torgrud, L. J., \& Evers, B. H. (1988). Effects of prior response-contingent reinforcement on superstitious behavior. Animal Learning \& Behavior, 16, 277-284.

Guthrie, E. R., \& Horton, G. P. (1946). Cats in a puzzle box. New York: Rinehart.

HAILMAN, J. P. (1982). Ontogeny: Toward a general theoretical framework for ethology. In P. P. G. Bateson \& P. H. Klopfer (Eds.), Perspectives in ethology (Vol. 5, pp. 133-189). New York: Plenum.

HANSON, S. J. (1991). Behavioral diversity, search and stochastic con- nectionist systems. In M. L. Commons, S. Grossberg, \& J. E. R. Staddon (Eds.), Quantitative analysis of hehavior: Neural network models of conditioning and action (pp. 295-344). Hillsdale. NJ: Erlbaum. innis, N. K., Simmelhag-Grant, V. L., \& Staddon. J. E. R. (1983). Behavior induced by periodic food delivery: The effects of interfood interval. Journal of the Experimental Analysis of Behavior, 39, 309322.

Justice, T. C., \& LoONey, T. A. (1990). Another look at "superstitions" in pigeons. Bulletin of the Psychonomic Society, 28, 64-66.

KAPLAN, H. L. ( 1983). Correlations, contrasts, and components: Fourier analysis in a more familiar terminology. Behavior Research Methods \& Instrumentation, 15, 228-241.

KILLEEN, P. (1975). On the temporal control of behavior. Psychological Review, 82, 89-115.

KILLEEN, P. (1979). Arousal: Its genesis, modulation, and extinction. In M. D. Zeiler \& P. Harzem (Eds.), Advances in analysis of behaviour: Vol. I. Reinforcement and the organization of behaviour (pp. 31-78). New York: Wiley.

MACHADO, A. (1989). Operant conditioning of behavioral variability using a percentile reinforcement schedule. Journal of the Experimental Analysis of Behavior, 52, 155-166.

MACHADO, A. (1992). Behavioral variability and frequency-dependent selection. Journal of the Experimental Analysis of Behavior, 58, 241. 263.

MACHADO, A. (1993). Learning variable and stereotypical sequences of responses: Some data and a new model. Behavioural Processes, 30 , 103-130.

MaCHADO, A. (1994). Polymorphic response patterns under frequencydependent selection. Animal Learning \& Behavior, 22, 53-71.

Mason, G. A. (1991a). Stereotypies: A critical review. Animal Behaviour, 41, 103-115.

Mason, G. A. (1991b). Stereotypies and suffering. Behavioural Processes, 25, 1015-1037.

MatThews, T. J., Bordi, F., \& Depollo, D. (1990). Scheduled-induced kinesis and taxic behavioral stereotypy in the pigeon. Journal of Experimental Psychology: Animal Behavior Processes, 16, 335-344.

MCElroy, E., \& NeUringer, A. (1990). Effects of alcohol on reinforced repetitions and reinforced variations in rats. Psychopharmacology, 102, 49-55.

Morgan, L., \& Neuringer, A. (1990). Behavioral variability as a function of response topography and reinforcement contingency. Animal Learning \& Behavior, 18, 257-263.

MORRIS, C. J. (1987). The operant conditioning of response variability: Free-operant versus discrete-response procedures. Journal of the Experimental Analysis of Behavior, 47, 273-277.

NEURINGER, A. (1991). Operant variability and repetition as functions of interresponse time. Journal of Experimental Psychology: Animal Behavior Processes, 17, 3-12.

Notterman, J. M., \& MinTZ, D. E. (1965). Dynamics of a response. New York: Wiley.

PAGE, S., \& NeURINGer, A. (1985). Variability is an operant. Journal of Experimental Psychology: Animal Behavior Processes, 11, 429-452.

PEAR, J. J. (1985). Spatiotemporal patterns of behavior produced by variable-interval schedules of reinforcement. Journal of the Experimental Analysis of Behavior, 44, 217-231.

PEAR, J. J. (1994). Problems and pitfalls for Killeen's principles of reinforcement. Behavioral \& Brain Sciences, 17, 146-147.

Pear, J. J., \& Eldridge, G. D. (1984). The operant-respondent distinction: Future directions. Journal of the Experimental Analysis of Behavior, 42, 453-467.

Pear, J. J., \& Legris, J. A. (1987). Shaping by automated tracking of an arbitrary operant response. Journal of the Experimental Analysis of Behavior, 47, 241-247.

Pisacreta, R. (1982). Some factors that influence the acquisition of complex, stereotyped, response sequences in pigeons. Journal of the Experimental Analysis of Behavior, 33, 359-369.

Press, W. P., Flannery, B. P., Teukolsky, S. A., \& Vetterling, W. T. (1989). Numerical recipes in Pascal: The art of scientific computing. Cambridge: Cambridge University Press.

Recuerda, P., Arias de Reyna, L., Redondo, T., \& Trujillo, J. (1987). Analyzing stereotypy in red deer alarm postures by means of information redundancy. Behavioural Processes, 14, 71-87. 
SCHWARTZ, B. (1980). Development of complex, stereotyped behavior in pigeons. Journal of the Experimental Analysis of Behavior, 33, 153-166.

ScHWARTZ, B. (1981). Reinforcement creates behavioral units. Behaviour Analysis Letters, 1, 33-41.

SCHWARTZ, B. (1982). Interval and ratio reinforcement of a complex sequential operant in pigeons. Journal of the Experimental Analysis of Behavior, 37, 349-357.

SEeley, R. J., \& Brozoski, T. J. (1989). Measurement and quantification of stereotypy in freely behaving subjects: An information analysis. Behavior Research Methods, Instruments, \& Computers, 21, 271274.

Silva, F. J., Pear, J. J., Tait, R. W., \& Forest, J. J. (in press). Fourier analysis of movement patterns in pigeons. Behavior Research Methods, Instruments, \& Computers.

Silva, F. J., Silva, K. M., \& PEAR, J. J. (1992). Sign- vs. goal-tracking: The effects of conditioned-stimulus-to-unconditioned-stimulus distance. Journal of the Experimental Analysis of Behavior, 57, 17-31.

SkINNER, B. F. (1938). The behavior of organisms: An experimental analysis. New York: Appleton-Century.

SKINNER, B. F. (1948). "Superstition" in the pigeon. Journal of the Experimental Psychology, 38, 168-172.

STADDON, J. E. R. (1992). The "superstition" experiment: A reversible figure. Journal of Experimental Psychology: General, 121, 270-272.

Staddon, J. E. R., \& Simmelhag, V. L. (1971). The "superstition" experiment: A reexamination of its implications for the principles of adaptive behavior. Psychological Review, 78, 3-43.

STEINBERG, J. B. (1977). Information theory as an ethological tool. In B. A. Hazlett (Ed.), Quantitative methods in the study of animal behavior (pp. 47-74). New York: Academic Press.

Terlouw, E. M. C., Lawrence, A. B., Ladewig, J., De Passille, A. M., Rushen, J., \& Schouten, W. G. P. (1991). Relationship between plasma cortisol and stereotypic activities in pigs. Behavioural Processes, 25, 133-153.

TIMBERLAKE, W. (1983). The functional organization of appetitive behavior: Behavior systems and learning. In M. D. Zeiler \& P. Harzem (Eds.), Advances in analysis of behaviour: Vol. 3. Biological factors in learning (pp. 177-221). Chichester, U.K.: Wiley.
TimberlaKe, W. (1990). Natural learning in the laboratory. In D. A Dewsbury (Ed.), Contemporary issues in comparative psychology (pp. 31-54). Sunderland, MA: Sinauer.

Timberlake, W., \& LuCAS, G. A. (1985). The basis of superstitious behavior: Chance contingency, stimulus substitution, or appetitive behavior? Journal of the Experimental Analysis of Behavior, 44, 279 299.

Timberlake, W., \& Silva, K. M. (1995). Appetitive behavior in ethology, psychology, and behavior systems. In N. Thompson (Ed.), Perspectives in ethology: Vol. 2. Behavioral design (pp. 211-253). New York: Plenum.

Vetterling, W. T., Teukolsky, S. A., Press, W. H., \& Flannery, B. P. (1989). Numerical recipes example book (Pascal) (rev. ed.). New York: Cambridge University Press.

VoGEL, R., \& ANNAU, Z. (1973). An operant discrimination task allowing variability of reinforced response patterning. Journal of the Experimental Analysis of Behavior, 20, 1-6.

WASSERMAN, E. A. (1981). Response evocation in autoshaping: Contributions of cognitive and comparative-evolutionary analyses to an understanding of directed action. In C. M. Locurto, H. S. Terrace, \& J. Gibbon (Eds.), Autoshaping and conditioning theory (pp. 21-54). New York: Academic Press.

Weber, B. H., DePeW, D. J., \& Smith, J. D. (1988). Entropy, information, and evolution: New perspectives on physical and biological evolution. Cambridge, MA: MIT Press.

\section{NOTE}

1. The description of Fourier analysis that is presented here is summarized from Kaplan's (1983) excellent user-friendly description of Fourier analysis, and we direct those seeking additional information to that article. For mathematically oriented descriptions of Fourier analysis, see Bloomfield (1976), Brockwell and Davis (1987), and Press et al (1989).

(Manuscript received February 2, 1994; revision accepted for publication September 19, 1994.) 